\title{
A SMALL ARITHMETIC HYPERBOLIC THREE-MANIFOLD
}

\author{
TED CHINBURG
}

\begin{abstract}
The hyperbolic three-manifold which results from $(5,1)$ Dehn surgery on the complement of a figure-eight knot in $S^{3}$ is arithmetic.
\end{abstract}

I. Introduction. Let $M$ be the complete orientable hyperbolic three-manifold which results from $(5,1)$ Dehn surgery on the complement of the figure-eight knot $K$ in $S^{3}$. In this note we will prove

THEOREM 1. $M$ is arithmetic.

A precise description of $M$ as an arithmetic manifold is given in the summary at the end of this paper. One consequence of Theorem 1 and the results of Borel in $[\mathbf{1}]$ is that

$$
\operatorname{Volume}(M)=12 \cdot 283^{3 / 2}{ }_{\zeta k}(2)(2 \pi)^{-6},
$$

where $\zeta_{k}(s)$ denotes the Dedekind zeta function of the unique quartic field $k$ of discriminant -283. Another consequence of Theorem 1 and Borel's work is that there exist infinitely many minimal elements in the set of manifolds commensurable to $M$.

By work of Jørgenson and Thurston (see $[7, \S 6.6]$ ), the set of volumes of complete orientable hyperbolic three-manifolds is a well-ordered subset of $\mathbf{R}$ of order type $\omega^{\omega}$. In particular, there is a minimal element $v_{1}$ in this set. In [4] Meyerhoff conjectured that $M$ has volume $v_{1}$, but this is shown to be not true by Weeks [9]. Weeks proved that the manifold $M^{\prime}$ obtained by $(5,1),(5,2)$ Dehn surgery on the complement of the Whitehead link in $S^{3}$ has

$$
\operatorname{Volume}\left(M^{\prime}\right)=0.9427 \ldots<\operatorname{Volume}(M)=0.9812 \ldots
$$

The author and Jørgenson have proved that Weeks' manifold $M^{\prime}$ is also arithmetic (to appear), but the minimal volume $v_{1}$ remains unknown. For further discussion of the volumes of hyperbolic three-manifolds and orbifolds, see Thurston $[\mathbf{7}, \mathbf{8}]$, Milnor [5], Borel [1], and Chinburg and Friedman [2].

\section{Proof of Theorem 1.}

LEMMA 1. The fundamental group $\pi_{1}(M)$ is generated by two elements $\bar{\alpha}$ and $\bar{\beta}$, which are subject to the relations $f(\bar{\alpha}, \bar{\beta})=g(\bar{\alpha}, \bar{\beta})=1$, where

$$
\begin{gathered}
f(a, b)=\left(a b^{-1} a^{-1} b\right) a\left(a b^{-1} a^{-1} b\right)^{-1} b^{-1}, \\
g(a, b)=a^{4} b^{-1} a b a^{-2} b a b^{-1} a .
\end{gathered}
$$

Received by the editors April 15, 1984 and, in revised form, March 6, 1986.

1980 Mathematics Subject Classification (1985 Revision). Primary 51M10, 57S30; Secondary 30F40, $12 A 99$.

Partially supported by NSF grant MCS-82-43648 and by the Sloan Foundation. 


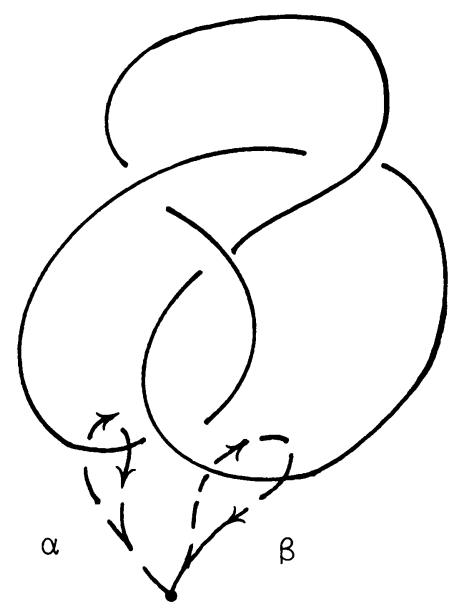

FIGURE 1

ProOF. Following Riley [6] and Milnor [5], we observe that $\pi_{1}\left(S^{3}-K\right)$ is generated by the two loops $\alpha$ and $\beta$ pictured in Figure 1, which are subject to the single relation $f(\alpha, \beta)=1$.

We take $\alpha$ as a meridian for a torus neighborhood $T$ of $K$ in $S^{3}$. A positively oriented longitude $l$ on $T$ is given by $\left(\alpha^{-1} \beta^{-1} \alpha\right) \beta \alpha^{-1}\left(\alpha^{-1} \beta \alpha \beta^{-1} \alpha\right)$. By the definition of hyperbolic Dehn surgery (cf. Thurston [7]). $\pi_{1}(M)$ is isomorphic to the quotient of $\pi_{1}\left(S^{3}-K\right)$ by the additional relation $\alpha^{5} l=g(\alpha, \beta)=1$.

LEMMA 2. There is a representation $\rho: \pi_{1}(M) \rightarrow \mathrm{SL}_{2}(\mathbf{C})$ with the following properties. The induced projective representation $\bar{\rho}: \pi_{1}(M) \rightarrow \mathrm{PSL}_{2}(\mathbf{C})$ is discrete and faithful, and $M$ is isometric to $H^{3} / \bar{\rho}\left(\pi_{1}(M)\right)$. Let $A=\rho(\bar{\alpha})$ and $B=\rho(\bar{\beta})$. There are nonzero $\lambda, \xi, r \in \mathbf{C}$ such that $|\lambda| \neq 1 \neq|\xi|$ and

$$
A=\left[\begin{array}{cc}
\lambda & 1 \\
0 & \lambda^{-1}
\end{array}\right] \quad \text { and } \quad B=\left[\begin{array}{cc}
\xi & 0 \\
r & \xi^{-1}
\end{array}\right] \text {. }
$$

Proof. Since $M$ is an orientable hyperbolic three-manifold, $M$ is isometric to $H^{3} / \rho_{1}\left(\pi_{1}(M)\right)$ for some discrete faithful representation $\rho_{1}: \pi_{1}(M) \rightarrow \operatorname{PSL}_{2}(\mathbf{C})$. Let $A$ and $B$ in $\operatorname{SL}_{2}(\mathbf{C})$ have images $\bar{A}=\rho_{1}(\bar{\alpha})$ and $\bar{B}=\rho_{1}(\bar{\beta})$ in $\operatorname{PSL}_{2}(\mathbf{C})$. Let $I$ be the $2 \times 2$ identity matrix. Then $f(A, B)= \pm I$ and $g(A, B)= \pm I$. By multiplying $A$ by \pm 1 and $B$ by \pm 1 , we may make $f(A, B)=g(A, B)=I$, so that $A$ and $B$ give a representation $\rho_{2}: \pi_{1}(M) \rightarrow \mathrm{SL}_{2}(\mathrm{C})$ lifting $\rho_{1}$. Because $M$ is compact (cf. Thurston [7]), $A$ and $B$ are hyperbolic.

Suppose that $A$ and $B$ have a common nonzero eigenvector. Then $(A B)^{-1} B A$ is unipotent. Since $\pi_{1}(M)$ can have no nontrivial parabolic elements, this would imply $(\bar{\alpha} \bar{\beta})^{-1} \bar{\beta} \bar{\alpha}=1$, contradicting the fact that $\pi_{1}(M)$ is nonabelian. Hence $A$ and $B$ have no common nonzero eigenvector.

We may now find a basis $\left\{v_{1}, v_{2}\right\}$ for $\mathbf{C}^{2}$ such that $A v_{1}=\lambda v_{1}$ and $B v_{2}=\xi^{-1} v_{2}$ for some nonzero $\lambda, \xi \in \mathbf{C}$ such that $|\lambda| \neq 1 \neq|\xi|$. Since $v_{2}$ is not an eigenvector of $A$, we may multiply $v_{1}$ by a nonzero scalar to have $A v_{2}=\lambda^{-1} v_{2}+v_{1}$. Relative to the basis $\left\{v_{1}, v_{2}\right\}, A$ and $B$ now have the form in (3) for some $r \in \mathbf{C}$, where $r \neq 0$ since 
$v_{1}$ is not an eigenvector of $B$. The resulting representation $\rho: \pi_{1}(M) \rightarrow \mathrm{SL}_{2}(\mathrm{C})$ is conjugate to $\rho_{2}$ in $\mathrm{SL}_{2}(\mathbf{C})$, and thus has the properties required.

LEMMA 3. The numbers $\lambda, \xi$, and $r$ of Lemma 2 are algebraic integers, and $\lambda=\xi$. The minimal polynomial of $r$ is $Q(z)=z^{4}-8 z^{3}+22 z^{2}-25 z+11$. There is a root $\eta$ of $P(z)=z^{4}+z^{3}-1$ such that $r=1-\eta /\left(\eta^{2}-1\right)$ and $\xi^{2}+\gamma \xi+1=0$, where $\gamma=\eta^{2}-1$.

Proof. The equation $f(A, B)=I$ is equivalent to $D A=B D$, where $D=$ $A B^{-1} A^{-1} B$. Compare the entries in the first row of $D A$ to those in the first row of $B D$. Since these entries cannot both be zero, we find that one of the following is true:

$$
\begin{array}{ccc}
\lambda=\xi^{-1} & \text { and } & \left(\xi^{-2}-r\right)\left(\xi^{2}+r\right)+r=0, \quad \text { or } \\
\lambda=\xi & \text { and } \quad(r-1)\left(2-r-\xi^{2}-\xi^{-2}\right)=1 .
\end{array}
$$

Let $c_{i, j}$ be the $(i, j)$ entry in $g(A, B)=I$. A computation shows that $c_{2,1}=0$ and (4) imply that either $\xi=1$ or $r=0$. Both of these possibilities are excluded by Lemma 2, so (5) must be true. To arrive at a polynomial equation $r$ must satisfy, one may write out the terms of $c_{1,1}^{2}=1$ and use (5) to eliminate the appearance of $\lambda$ and $\xi$. One finds that $r$ must be a root of $(z-1)^{7}\left(z^{2}-z+1\right) Q(z)^{2}$, where $Q(z)$ is as in the statement of Lemma 3. Clearly (5) implies $r \neq 1$, while if $r^{2}-r+1=0$ then (5) implies $|\xi|=1$, contradicting Lemma 2. Hence $r$ is a root of $Q(z)$.

Elementary calculations show that $r=1-\eta /\left(\eta^{2}-1\right)$ for some root $\eta$ of the irreducible polynomial $P(z)=z^{4}+z^{3}-1$. From (5) we have $\left(\xi+\xi^{-1}\right)^{2}=4-$ $r-1 /(r-1)=\gamma^{2}$, where $\gamma=\eta^{2}-1$. Hence $\xi+\xi^{-1}= \pm \gamma$. Calculation shows $g(A, B)=-I$ if $\xi+\xi^{-1}=\gamma$, so we must have $\xi+\xi^{-1}=-\gamma$ and the lemma is proved.

We will now prove Theorem 1.

With the notations of Lemmas 2 and 3, let $\mathbf{B}$ be the vector space over the field $k=\mathbf{Q}(\eta)$ with basis $\{I, A, B, A B\}$. We have $A^{2}+\gamma A+I=B^{2}+\gamma B+I=$ $(A B)^{2}+\left(\gamma^{2}-2+r\right) A B+I=0$. Since $\gamma$ and $r$ are in $k$, one checks that $\mathbf{B}$ is a quaternion algebra over $k$. There are two real places of $k$, and no nonzero solutions $(x, y, z) \in \mathbf{R}^{3}$ to the equation $\operatorname{det}(x I+y A+z B)=0$ when $A$ and $B$ are embedded into $M_{2}(\mathbf{C})$ via an embedding over either real place of $k$. Hence $\mathbf{B}$ is ramified at the two real places of $k$.

Let $F$ be the field $k(\xi)$, and let $\underline{0}_{F}$ be the integers of $F$. By Lemma $3, \mathbf{B}$ splits over $F$. Let $D$ be the maximal order $\mathbf{B} \cap M_{2}\left(\underline{0}_{F}\right)$ in $\mathbf{B}$, and let $D^{1}=\mathbf{B} \cap \mathrm{SL}_{2}\left(\underline{0}_{F}\right)$. The field $k$ has a unique complex place $\infty$. The completion $k_{\infty}$ of $k$ at $\infty$ is isomorphic to $\mathbf{C}$, and we have an isomorphism $f_{\infty}: \mathbf{B} \otimes_{k} k_{\infty} \stackrel{\sim}{\rightarrow} M_{2}(\mathbf{C})$.

Define $\Gamma_{D}^{1}$ to be the image of $f_{\infty}\left(D^{1}\right) \subseteq \mathrm{SL}_{2}(\mathbf{C})$ in $\mathrm{PSL}_{2}(\mathbf{C})$. In [1] Borel proves that $\Gamma_{D}^{1}$ is a discrete subgroup of $\mathrm{PSL}_{2}(\overline{\mathbf{C}})$, and that

$$
\operatorname{Volume}\left(H^{3} / \Gamma_{D}^{1}\right)=\prod_{v \in R_{f}}(N v-1)\left|D_{k}\right|^{3 / 2} \zeta_{k}(2)(2 \pi)^{-6} .
$$

Here $R_{f}$ denotes the set of finite places of $k$ where $\mathbf{B}$ is ramified, and $N v$ is the norm of the finite place $v$. The discriminant $D_{k}$ of $k$ is -283 , and $\zeta_{k}(s)$ denotes the Dedekind zeta function of $k$. 
From Lemmas 2 and 3 , we have $\bar{\rho}\left(\pi_{1}(M)\right) \subseteq \Gamma_{\mathcal{D}}^{1}$. We may now use the trivial estimate $\zeta_{k}(2) \geq 1$ in (6) and Meyerhoff's estimate Volume $(M)<1$ in $([4],[8$, p. 365]) to deduce that

$$
\begin{aligned}
1 \leq\left[\Gamma_{D}^{1}: \bar{\rho}\left(\pi_{1}(M)\right)\right] & \leq \prod_{v \in R_{f}}(N v-1)^{-1}\left|D_{k}\right|^{-3 / 2}(2 \pi)^{6} \\
& \leq 13 \prod_{v \in R_{f}}(N v-1)^{-1}
\end{aligned}
$$

A quaternion algebra over $k$ is determined by the even number of places where it ramifies. In particular, there are an even number of places in $R_{f}$. The discriminant of the polynomial $P(z)=z^{4}+z^{3}-1$ in Lemma 3 is -283 , and $P(z)$ is irreducible modulo 2 and 3 . Hence the rational primes 2 and 3 are inert in $k$. One may now check that $\# R_{f}$ even and $\prod_{v \in R_{f}}(N v-1) \leq 13$ imply that $R_{f}$ is empty. The algebra $\mathbf{B}$ must therefore be isomorphic to $H_{\mathbf{Q}} \otimes_{\mathbf{Q}} k$, where $H_{\mathbf{Q}}$ denotes the Hamilton quaternion algebra over $\mathbf{Q}$.

Let $v_{11}$ be the first-degree unramified place of $k$ corresponding to the prime ideal $r \underline{0}_{k}$ lying over the rational prime 11 . From the equation $\xi^{2}+\gamma \xi+1=0$ we find that $v_{11}$ splits into two places $v_{11}^{\prime}$ and $v_{11}^{\prime \prime}$ in $F=k(\xi)$. Let $\Gamma_{11}$ be the group of $\left(\begin{array}{ll}a & b \\ c & d\end{array}\right) \in M_{2}\left(\underline{0}_{F}\right)$ which are in $D^{1}$ and for which $c$ is a nonunit at $v_{11}^{\prime}$. Define $\Gamma_{0}\left(v_{11}^{\prime}\right)$ to be the image of $f_{\infty}\left(\Gamma_{11}\right) \subseteq \mathrm{SL}_{2}(\mathbf{C})$ in $\operatorname{PSL}_{2}(\mathbf{C})$.

Lemmas 2 and 3 show that $\bar{\rho}\left(\pi_{1}(M)\right) \subseteq \Gamma_{0}\left(v_{11}^{\prime}\right)$. Borel proves in [1, pp. 1314] that $\left[\Gamma_{D}^{1}: \Gamma_{0}\left(v_{11}^{\prime}\right)\right]=N v_{11}+1=12$. The fact, (7), and $R_{f}=\varnothing$ show that $\bar{\rho}\left(\pi_{1}(M)\right)=\Gamma_{0}\left(v_{11}^{\prime}\right)$. Since $H^{3} / \Gamma_{0}\left(v_{11}^{\prime}\right)$ is an arithmetic hyperbolic three-manifold, Theorem 1 now follows from Lemma 2.

The first statement in the following summary is shown by Godwin in [3].

SUMMARY. The field $k=\mathbf{Q}(\eta)$ generated by a root of $\eta^{4}+\eta^{3}-1=0$ is up to isomorphism the unique quartic field of discriminant -283 . The quaternion algebra $\mathbf{B}$ over $k$ is isomorphic to $H_{\mathbf{Q}} \otimes_{\mathbf{Q}} k$, where $H_{\mathbf{Q}}$ denotes the Hamilton quaternion algebra over $\mathbf{Q}$. Let $v_{11}^{\prime}$ be one of the two first-degree places over the rational prime 11 in the field $F=\mathbf{Q}(\xi)$ generated by a root of $\xi^{2}+\left(\eta^{2}-1\right) \xi+1=0$. The inclusion of $k$ into $F$ induces an injection $\mathbf{B} \rightarrow \mathbf{B} \otimes_{k} F \cong M_{2}(F)$. Let $\Gamma_{11}$ be the group of $\left(\begin{array}{ll}a & b \\ c & d\end{array}\right) \in \mathrm{SL}_{2}\left(\underline{0}_{F}\right)$ which are in $\mathbf{B}$ and for which $c$ is a nonunit at $v_{11}^{\prime}$, where $\underline{0}_{F}$ denotes the integers of $F$. Let $\Gamma_{0}\left(v_{11}^{\prime}\right)$ be the image of $\Gamma_{11}$ in $\mathrm{PSL}_{2}(\mathbf{C})=\mathbf{B}_{\infty}^{1} /\{ \pm I\}$ where $\mathbf{B}_{\infty}^{1}$ is the group of elements of reduced norm 1 in the completion $\mathbf{B}_{\infty} \cong M_{2}(\mathbf{C})$ of $\mathbf{B}$ at the unique complex place $\infty$ of $k$. Then $(5,1)$ Dehn surgery on the complement of a figure-eight knot in $S^{3}$ yields a hyperbolic manifold isometric to $H^{3} / \Gamma_{0}\left(v_{11}^{\prime}\right)$.

ACKNOWledgment. The author would like to thank R. Meyerhoff, J. Milnor, and $\mathrm{W}$. Thurston for useful conversations.

\section{REFERENCES}

1. A. Borel, Commensurability classes and volumes of hyperbolic 3-manifolds, Ann. Scuola Norm. Sup. Pisa Cl. Sci. (4) 8 (1981), 1-33.

2. T. Chinburg and E. Friedman, The smallest arithmetic hyperbolic three-orbifold, Invent. Math. (to appear). 
3. H. J. Godwin, On quartic fields of signature one with small discriminant, Quart. J. Math. Oxford 8 (1957), 214-222.

4. R. Meyerhoff, $A$ lower bound for the volume of hyperbolic 3-manifolds, preprint (1982).

5. J. Milnor, Hyperbolic geometry: The first 150 years, Bull. Amer. Math. Soc. (N.S.) 6 (1982) 9-24.

6. R. Riley, A quadratic parabolic group, Math. Proc. Cambridge Philos. Soc. 77 (1975), 281-288.

7. W. Thurston, The geometry and topology of 3-manifolds, Princeton Univ. preprint (1978).

8. __ Three dimensional manifolds, Kleinian groups and hyperbolic geometry, Bull. Amer. Math. Soc. (N.S.) 6 (1982), 357-381.

9. J. Weeks, Hyperbolic structures on three-manifolds, Princeton $\mathrm{Ph} . \mathrm{D}$. thesis (1985).

Department of Mathematics, University of Pennsylvania, Philadelphia, PENNSYLVANIA 19104

Current address: Mathematical Sciences Research Institute, 1000 Centennial Drive, Berkeley, California 94720 